\title{
The Course of COVID-19 in a Patient with Aplastic Anemia and Paroxysmal Nocturnal Hemoglobinuria Clone: A Case Report and Literature Review
}

\author{
Sreethish Sasi ${ }^{a}$ Mohamed A. Yassin ${ }^{b}$ Arun P. Nair ${ }^{c}$ Afraa M. Fadul ${ }^{b}$ \\ Mohammed A. Abukhattab ${ }^{c}$ \\ aDepartment of Internal Medicine, Hamad General Hospital, Hamad Medical Corporation, Doha, Qatar; \\ ${ }^{b}$ Department of Hematology, National Center for Cancer Care and Research, Hamad Medical Corporation, \\ Doha, Qatar; 'Department of Infectious Diseases, Communicable Diseases Center, Hamad Medical Corporation, \\ Doha, Qatar
}

\section{Keywords}

Aplastic anemia · Paroxysmal nocturnal hemoglobinuria · COVID-19 $\cdot$ Medicine $\cdot$ Hematology

\begin{abstract}
Introduction: Aplastic anemia (AA) and paroxysmal nocturnal hemoglobinuria $(\mathrm{PNH})$ are bone marrow failure syndromes. A $20-40 \%$ of patients with AA have a PNH clone at diagnosis. To date, there are little data about the course of COVID-19 in patients with AA and PNH. Case Presentation: A 36-year-old gentleman, who was previously diagnosed as a case of AA with PNH clones off immune-suppressive therapy, presented with fever and cough and was diagnosed with mild pneumonia due to COVID-19 with positive nasopharyngeal swab polymerase chain reaction (PCR) for severe acute respiratory syndrome coronavirus 2 . His clinical course was benign except transient thrombocytopenia. He was asymptomatic after day 4, and viral PCR was negative on day 21. Discussion: Though studies have shown that COVID-19 is associated with lymphopenia, our patient had a normal to high lymphocyte count. The neutrophil to lymphocyte ratio (NLR) was $<1$ during COVID-19, which correlates with the
\end{abstract}

karger@karger.com www.karger.com/dmj

Karger $\stackrel{\text { ' }}{5}$
(C) 2021 The Author(s)

Published by S. Karger AG, Basel

This is an Open Access article licensed under the Creative Common Attribution-NonCommercial-4.0 International License (CC BY-NC) (http://www.karger.com/Services/OpenAccessLicense), applicable to the online version of the article only. Usage and distribution for commercial purposes requires written permission. mild course of the disease. To know whether elevated lymphocyte count, low NLR, and benign course of COVID-19 is a standard feature for all patients with underlying $A A$, we need more case reports and series. The significance of this case report is that it describes the course of COVID-19 in a patient with $\mathrm{AA}$ and $\mathrm{PNH}$ clones and, up to our knowledge, is the first report showcasing the association between these rare combinations of diseases.

(C) 2021 The Author(s).

Published by S. Karger AG, Basel

\section{Introduction}

Aplastic anemia (AA) is a myelosuppressive disorder with no hematopoietic stem cells in the bone marrow. Pancytopenia is a characteristic feature of AA, and many cases occur in conjunction with a paroxysmal nocturnal hemoglobinuria $(\mathrm{PNH})$ clone [1]. $\mathrm{PNH}$ is an infrequent, life-threatening hematopoietic disorder where the red blood cells are destroyed by the complement system. Mutation of the phosphatidylinositol $\mathrm{N}$-acetylglucosaminyltransferase subunit A (PIG-A) gene causes a deficiency of CD55 and CD59 anchoring proteins on the surface of red 
blood cells, which makes them susceptible to direct destruction by the complement system [2]. Two to 4 of every 10 newly diagnosed AA patients have a PNH clone [3]. $\mathrm{PNH}$ cells have a low capacity to anchor glycoproteins leading to reduced activity of CD58. CD58 plays a significant role in T-cell-mediated cytotoxicity, and their depletion makes the PNH cells resistant to autoimmunity [4]. Though COVID-19 has become a major medical emergency worldwide, there are little data available about its course in patients with AA. Patients on immunosuppression are considered to be at a higher risk for infection and complications of COVID-19. However, considering that it reduces the effects caused by destruction of tissues and inflammatory response, immunosuppression may prove beneficial during the course of the disease.

\section{Case Report/Case Presentation}

A 36-year-old gentleman presented to our emergency department with 2 days of sore throat, followed by 3 days of fever and cough. Fever was low grade without chills. Cough was productive with whitish sputum, but no hemoptysis or epistaxis. There was no breathing discomfort or chest pain. Bowel and bladder habits were regular, and no cola-colored urine was noticed. He had no history of exposure to any sick patients. Past history was significant for AA with PNH clones diagnosed 3 years back, when he presented with epistaxis and generalized purpuric rashes all over the body. At that time, peripheral blood showed pancytopenia (moderate normochromic normocytic anemia, mild neutropenia, and marked thrombocytopenia) and reticulocytopenia. Bone marrow aspirate and biopsy showed hypocellular bone marrow with decreased trilineage hematopoiesis, almost absent megakaryopoiesis, and no increase in blasts. Flow cytometry from the bone marrow aspirate showed $<0.5 \%$ of CD34-positive myeloblasts with no evidence of monotypic B-cell population, while flow cytometry analysis of peripheral blood showed a small PNH clone within the monocytes $(13 \%)$ and granulocytes $(11 \%)$ and a minute clone in the red cells $(0.7 \%)$. He was started on cyclosporine $125 \mathrm{mg}$ twice a day, which was reduced to $100 \mathrm{mg}$ after 4 months owing to side effects (hyperuricemia, gouty arthritis, and gum hypertrophy). The possibility of hematopoietic stem cell transplantation (HSCT) was thought about after 6 months of cyclosporin therapy as there was an inadequate response. But, a histocompatible donor was not available. Request to repeat the bone marrow examination after a year of treatment was refused by the patient. Flow cytometry from peripheral blood was repeated after 2 years of cyclosporin therapy, and it showed a large PNH clone within the monocytes (76\%), granulocytes (67\%), and red cells (43\%). The clone size was increased from the previous analysis. He had regular follow-ups with hematology and was last seen a month before the current presentation. The blood count at that point showed a hemoglobin of $12.7 \mathrm{~g}$ per dL, white cells of 4,900 per $\mu \mathrm{L}$, and platelets of $137 \times 103$ per $\mu \mathrm{L}$, all of which were stable over the past year (baseline), during which he was off immunosuppressive therapy (IST). The family history was unremarkable.

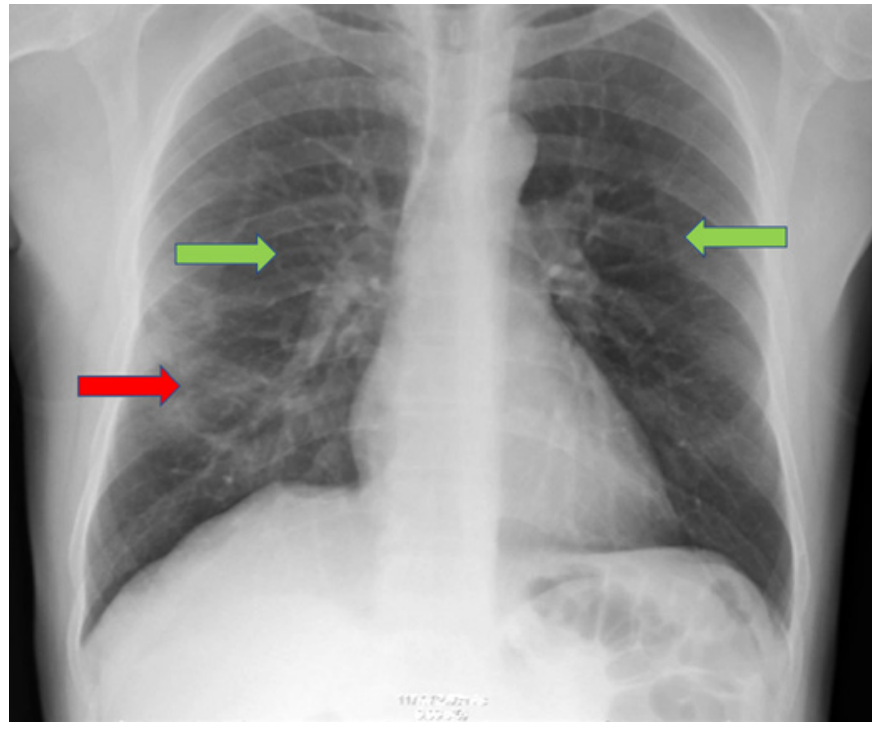

Fig. 1. Chest X-ray on admission. Chest X-ray on the day of admission showing a mild increase in bronchovascular markings bilaterally (green arrows) with a patchy area of haziness in the right lower zone (red arrow).

Physical examination during the current presentation was unremarkable except mildly congested throat. Blood pressure and heart rate were normal, and he was maintaining $100 \%$ saturation in room air. All systems were within normal limits. There were no bleeding manifestations. Urine dipstick was normal. Complete blood counts on admission showed mildly lower white cells $(3,900$ per $\mu \mathrm{L})$ and platelets $(99 \times 103$ per $\mu \mathrm{L})$ compared to his baseline. His laboratory testing results at baseline, at admission, during the hospital stay, and at discharge are shown in Table 1. The polymerase chain reaction (PCR) test for COVID-19 from a nasopharyngeal and throat swab was positive. Combined nasopharyngeal and oropharyngeal swabs (Copan Diagnostics Inc., Italy) were collected and placed in Universal Transport Medium. Aliquots of Universal Transport Medium were either extracted on the QIAsymphony platform (Qiagen, USA) and tested by reverse transcriptase (RT)-PCR with the Thermo Fisher TaqPath ${ }^{\mathrm{TM}} \mathrm{CO}-$ VID-19 RT-PCR Kit (Thermo Fisher, USA), targeting the S, N, and ORF-1a/b genes, or loaded directly on to a Roche Cobas ${ }^{\circledR} 6800$ and assayed with the Cobas ${ }^{\circledR}$ SARS-CoV-2 Test (Roche, Switzerland) targeting the ORF-1a/b and E-gene regions of SARS-CoV-2 [5]. Initial chest X-ray showed a mild increase in bronchovascular markings bilaterally with a patchy area of haziness in the right lower zone (Fig. 1). The QTc interval was $391 \mathrm{~ms}$, and he was started on treatment for mild COVID-19 pneumonia, as per our hospital protocol. He received azithromycin $500 \mathrm{mg}$ daily for 7 days, hydroxychloroquine $400 \mathrm{mg}$ twice a day for 1 day followed by daily for 9 days, oseltamivir $150 \mathrm{mg}$ twice a day for 5 days, and ceftriaxone $2 \mathrm{~g}$ daily for 7 days. His hospital stay was clinically uneventful, and he was never febrile. Laboratory results showed a significant drop in platelet count till day 15 and improvement after that. Repeated COVID-19 PCR was positive on day 14 but turned negative on days 21 and 22, and he was discharged for 2 weeks of home isolation. 
Table 1. Results of important laboratory tests at baseline, day 1, day 10, and day 21

\begin{tabular}{|c|c|c|c|c|c|}
\hline \multirow[t]{2}{*}{ Detail } & \multicolumn{4}{|l|}{ Result } & \multirow[t]{2}{*}{ Normal range } \\
\hline & baseline & day 1 & day 15 & day 22 & \\
\hline \multicolumn{6}{|l|}{ Complete blood count } \\
\hline White blood cells, $\times 10^{3} / \mu \mathrm{L}$ & 4.9 & 3.9 & 2.8 & 4.8 & $4-10$ \\
\hline Red blood cells, $\times 10^{6} / \mu \mathrm{L}$ & 3.9 & 3.9 & 3.8 & 4 & $4.5-5.5$ \\
\hline Platelets, $\times 10^{3} / \mu \mathrm{L}$ & 137 & 99 & 27 & 75 & $150-400$ \\
\hline Hemoglobin, g/dL & 12.7 & 12.4 & 12.1 & 12.8 & $13.0-17.0$ \\
\hline Hematocrit, $\%$ & 35.9 & 35.6 & 35.6 & 37.3 & $40.0-50.0$ \\
\hline Mean corpuscular volume, fL & 92.3 & 91.8 & 93.4 & 93.5 & $83.0-101.0$ \\
\hline Mean corpuscular hemoglobin, pg & 32.6 & 32 & 31.8 & 32.1 & $27.0-32.0$ \\
\hline Mean corpuscular hemoglobin concentration, g/dL & 35.4 & 34.8 & 34 & 34.3 & $31.5-34.4$ \\
\hline RDW, \% & 12.9 & 12.9 & 13.3 & 12.3 & $11.6-14.5$ \\
\hline Reticulocytes, \% & 1.4 & 1.2 & 0.9 & 1.1 & $0.5-2.5$ \\
\hline Absolute neutrophil count, $\times 10^{3} / \mu \mathrm{L}$ & 2.8 & 1.3 & 0.7 & 1.7 & $2-7$ \\
\hline Lymphocyte, $\times 10^{3} / \mu \mathrm{L}$ & 1.6 & 2.2 & 1.77 & 2.65 & $1-3$ \\
\hline Monocyte, $\times 10^{3} / \mu \mathrm{L}$ & 0.4 & 0.44 & 0.34 & 0.44 & $0.2-1$ \\
\hline Eosinophil, $\times 10^{3} / \mu \mathrm{L}$ & 0.0 & 0.0 & 0.0 & 0.0 & $0-0.5$ \\
\hline Basophil, $\times 10^{3} / \mu \mathrm{L}$ & 0.0 & 0.0 & 0.0 & 0.0 & $0-0.2$ \\
\hline \multicolumn{6}{|l|}{ Comprehensive metabolic panel } \\
\hline Urea, $\mathrm{mmol} / \mathrm{L}$ & 4.4 & 5.75 & 5.8 & 4.1 & $2.8-8.1$ \\
\hline Creatinine, $\mu \mathrm{mol} / \mathrm{L}$ & 86 & 90 & 98 & 85 & $62-106$ \\
\hline Total bilirubin, $\mu \mathrm{mol} / \mathrm{L}$ & 14 & 12.8 & 12 & 14.7 & $3.4-20.5$ \\
\hline Alkaline phosphatase, U/L & 70 & 85.8 & 80 & 60.7 & $40-150$ \\
\hline Alanine amino transferase, $\mathrm{U} / \mathrm{L}$ & 17 & 27.4 & 34 & 26 & $0-55$ \\
\hline Aspartate amino transferase, $\mathrm{U} / \mathrm{L}$ & 35 & 46 & 52 & 38 & $5-34$ \\
\hline C-reactive protein, $\mathrm{mg} / \mathrm{L}$ & - & 6 & 3.2 & - & $0-5$ \\
\hline
\end{tabular}

$\mu \mathrm{L}$, microliters; g/dL, grams/deciliter; fL, femtoliters; pg, picograms; mmol/L, millimoles per liter; $\mu \mathrm{mol} / \mathrm{L}$, micromoles per liter; U/L, International units per liter; mg/dL, milligrams per deciliter.

\section{Discussion/Conclusion}

Severe AA cases are treated with HSCT or IST. The most familiar agents used in IST are horse antithymocyte globulin (ATG), cyclosporine, and eltrombopag. HSCT has a high rate of cure and is the preferred mode of treatment in those $<40$ years of age with a histocompatible donor [6]. The reason our patient did not undergo HSCT is due to the unavailability of an HLA-matched donor. The COVID-19 pandemic poses stress on the health care system, including HSCT, IST, and cellular therapy, such as with CAR T cells [7]. According to the recommendations of the Infectious Diseases Society of America (IDSA), COVID-19 screening should be done universally before initiation of IST, even if not exposed [8]. An essential question during the COVID-19 pandemic is whether to continue definitive treatment for AA, thus exposing the patients to an increased risk of contracting SARS-CoV-2 or delaying the treatment. A systematic re- view of the associations between immune-suppressive and stimulating drugs and COVID-19 showed no evidence that specific cytotoxic drugs, low-dose methotrexate, JAK kinase inhibitors, or antitumor necrosis factor- $\alpha$ agents are contraindicated. So, routinely withholding critical anticancer or IST is not recommended for those who do not have COVID-19. Low-dose prednisolone and tacrolimus may have beneficial impacts, and the role of mycophenolate mofetil is less clear [9]. According to the American Society of Hematology, risks of delaying HSCT or IST outweigh the risks of exposure to the coronavirus in patients with an ANC $<200 / \mu \mathrm{L}$. HSCT may be restricted if ANC $>200 / \mu \mathrm{L}$ [7]. There is evidence that the herpes viral load increases after ATG treatment [10]. So, caution is needed as the same mechanism may extend to the SARS-CoV-2. Also, hospitalization of patients for ATG administration may be difficult due to limited beds and the risk of exposure. Severe viral infections are not expected with cyclosporine. Expectant care, with oral cyclo- 
sporine and eltrombopag, is recommended in patients with an ANC $>200 / \mu \mathrm{L}$ [7]. All transplant procedures should be delayed for at least 14 days in case the subject has a close exposure to a confirmed COVID-19 case [11]. A peculiar finding in our patient is that his neutrophil to lymphocyte ratio (NLR) throughout the course of COVID-19 was $<1$. Studies have shown that COVID-19 is associated with lymphopenia, and an elevated NLR correlates with the severity of the illness [12].

Even though patients with myelosuppression on or off chemotherapy are considered as high risk for serious complications of COVID-19, our patient had a relatively benign course. He had only mild pneumonia and never required respiratory support. Transient thrombocytopenia was the only major complication. The mild course of illness in our patient correlates with his low NLR score. However, the fact that his lymphocyte count remained normal to high throughout the course of disease is unusual of COVID-19. There is a case report from China, which has also noted normal to elevated lymphocyte counts in an AA patient with COVID-19 [13]. To know whether normal to high lymphocyte count, low NLR, and benign course of COVID-19 is a common feature for all patients with underlying $\mathrm{AA}$, we need more case reports and series.

\section{Acknowledgment}

The authors would like to acknowledge the Internal Medicine Residency Program of the Hamad Medical Corporation for scientific support.

\section{Statement of Ethics}

The case was approved by the Medical Research Center at Hamad Medical Corporation. A written informed consent was given by the patient to publish his case information and details.

\section{Conflict of Interest Statement}

The authors have no conflicts of interest to declare.

\section{Funding Sources}

The publication of this article was funded by the Qatar National Library.

\section{Author Contributions}

S.S.: manuscript preparation and literature search. He will act as a study guarantor. M.A.Y.: patient management, manuscript review and editing, and mentor. A.P.N.: patient management and manuscript review. A.F.M.: patient management and manuscript review. M.A.A.: patient management and manuscript review.

\section{Data Availability Statement}

All data generated or analyzed during this study are included in this article. Further enquiries can be directed to the corresponding author.

\section{References}

1 Young NS. Hematopoietic cell destruction by immune mechanisms in acquired aplastic anemia. Semin Hematol. 2000;37(1):3-14

2 Cappellini MD. Coagulation in the pathophysiology of hemolytic anemias. Hematology Am Soc Hematol Educ Program. 2007:748

3 Parker C, Omine M, Richards S, Nishimura J, Bessler M, Ware R, et al. Diagnosis and management of paroxysmal nocturnal hemoglobinuria. Blood. 2005;106(12):3699-709.

4 Young NS. The problem of clonality in aplastic anemia: Dr Dameshek's riddle, restated. Blood. 1992;79(6):1385-92.

5 Sasi S, Yassin MA, Nair AP, Al Maslamani MS. A case of COVID-19 in a patient with asymptomatic hemoglobin D thalassemia and glucose-6-p; hosphate dehydrogenase deficiency. Am J Case Rep. 2020;21:e925788.

6 Scheinberg P, Young NS. How I treat acquired aplastic anemia. Blood. 2012;120(6): 1185-96.
7 COVID-19 and Aplastic Anemia - Hematology.org [Internet]. [cited 2020 May 26]. Available from: https://www.hematology.org: 443/covid-19/covid-19-and-aplastic-anemia.

8 Infectious Diseases Society of America Guidelines on the Diagnosis of COVID-19 [Internet]. [cited 2020 May 26]. Available from: https: //www.idsociety.org/practice-guideline/covid-19-guideline-diagnostics/.

9 Russell B, Moss C, George G, Santaolalla A, Cope A, Papa S, et al. Associations between immune-suppressive and stimulating drugs and novel COVID-19: a systematic review of current evidence. Ecancermedicalscience. 2020;14:1022.

10 Olson AL, Dahi PB, Zheng J, Devlin SM, Lubin $M$, Gonzales AM, et al. Frequent human herpesvirus-6 viremia but low incidence of encephalitis in double-unit cord blood recipients transplanted without antithymocyte globulin. Biol Blood Marrow Transplant. 2014;20(6):787-93.
11 Ljungman P, Mikulska M, de la Camara R, Basak GW, Chabannon C, Corbacioglu S, et al. The challenge of COVID-19 and hematopoietic cell transplantation; EBMT recommendations for management of hematopoietic cell transplant recipients, their donors, and patients undergoing CAR T-cell therapy [published online ahead of print, 2020 May 13]. Bone Marrow Transplant. 2020;55(11):20716.

12 Yang AP, Liu JP, Tao WQ, Li HM. The diagnostic and predictive role of NLR, d-NLR and PLR in COVID-19 patients. Int Immunopharmacol. 2020;84:106504.

$13 \mathrm{Wu}$ T, Kang SC, Feng W, Fu H, Zhu XH, Wang XJ, et al. [A case report of aplastic anemia accompanied with COVID-19]. Zhonghua Xue Ye Xue Za Zhi. 2020;41(4):340. 